\title{
BROMOCEA Code: An Improved Grand Canonical Monte Carlo/Brownian Dynamics Algorithm Including Explicit Atoms
}

\author{
Carlos J. F. Solano, Karunakar R. Pothula, Jigneshkumar D. Prajapati, \\ Pablo M. De Biase, Sergei Yu. Noskov and Ulrich Kleinekathöfer
}

\section{Contents}

1 Nonbonded terms 1

1.1 Electrostatic and original core-repulsive steric potentials . . . . . . . . . . . . 1

1.2 Reaction field potential . . . . . . . . . . . . . . . . . . 3

1.2.1 Generalized basis-set expansion . . . . . . . . . . . . . . 3

1.2.2 Generalized Born model . . . . . . . . . . . . . . . . . . . . . . 6

2 SHAKE algorithm $\quad 8$

3 Random number generator $\quad 9$

4 Additional results from GCMC/BD simulations $\quad 10$

5 Molecular Dynamics Simulations $\quad 16$

\section{Nonbonded terms}

To improve the performance in the computation of nonbonded terms, we store precalculated values of the electrostatic, reaction field and original core-repulsive steric potentials in a system of look-up tables which are subsequently used during the GCMC/BD simulations. In the BROMOCEA code, we have also implemented some additional improvements for increasing the computational efficiency. Thereby, a more efficient implementation of the 2nd-order and 3rdorder B-splines functions for interpolating the values from previous look-up tables is included. In addition, more stable and efficient recurrence relations are employed in the calculation of real spherical harmonics required in the generalized basis-set expansion of the reaction field. In this section, we describe some of these new features in detail. For the sake of completeness, we also include a brief description of some complementary topics.

\subsection{Electrostatic and original core-repulsive steric potentials}

The electrostatic potential from protein charges and transmembrane potential is obtained by solving the modified Poisson-Boltzmann equation [1] on a discrete grid using standard finitedifference relaxation methods [2]. Let $N_{x}, N_{y}$ and $N_{z}$ denote the numbers of grid points in the $x, y$ and $z$ axes, respectively. Moreover, $\left(x_{a}^{c}, y_{b}^{c}, z_{c}^{c}\right)$ and $h$ denote the center grid point and 
the grid spacing, respectively. Thereby, the grid points $\left(x_{a}, y_{b}, z_{c}\right)$ are defined in the respective intervals $x_{a} \in\left[x_{a}^{c}-\left(N_{x}-1\right) h / 2, x_{a}^{c}+\left(N_{x}-1\right) h / 2\right], y_{b} \in\left[y_{b}^{c}-\left(N_{y}-1\right) h / 2, y_{b}^{c}+\left(N_{y}-1\right) h / 2\right]$ and $z_{c} \in\left[z_{c}^{c}-\left(N_{z}-1\right) h / 2, z_{c}^{c}+\left(N_{z}-1\right) h / 2\right]$. For a simplified implementation, one defines the scaled grid points $x_{a}^{n}=x_{a}-x_{a}^{c}+\left(N_{x}-1\right) h / 2, y_{b}^{n}=y_{b}-y_{b}^{c}+\left(N_{y}-1\right) h / 2$ and $z_{c}^{n}=z_{c}-z_{c}^{c}+\left(N_{z}-1\right) h / 2$ so that

$$
\left\{\begin{aligned}
x_{a}^{n}=h a ; & a=0, \ldots, N_{x}-1 \\
y_{b}^{n}=h b ; & b=0, \ldots, N_{y}-1 \\
z_{c}^{n}=h c ; & c=0, \ldots, N_{z}-1 .
\end{aligned}\right.
$$

In this approximation, the shielded static external potential resulting from the permanent protein charge distribution and the transmembrane potential at the position of particle $i$ is given by

$$
\phi_{s f}^{m}\left(\mathbf{r}_{i}\right)=\sum_{a, b, c} M_{m}(x) M_{m}(y) M_{m}(z) \phi_{s f}(a, b, c)
$$

where $\phi_{s f}(a, b, c)$ is the potential value at the grid point $\left(x_{a}^{n}, y_{b}^{n}, z_{c}^{n}\right)$ and $M_{m}(\zeta)$ denotes $m$ thorder B-spline functions $(m=2,3)$. The 2 nd-order B-spline function $M_{2}(\zeta)=1-|\zeta-1|$ is given by the continuous function

$$
M_{2}(\zeta)=\left\{\begin{aligned}
\zeta, & 0 \leq \zeta \leq 1 \\
2-\zeta, & 1 \leq \zeta \leq 2
\end{aligned}\right.
$$

where $\zeta=\{x, y, z\}$ satisfies $0 \leq \zeta \leq 2$. According to this relation, $\zeta$ is defined as $\zeta=1+\left(\zeta_{g}^{n}-\zeta_{i}^{n}\right) / h$ with $\zeta_{g}^{n}=\left\{x_{a}^{n}, y_{b}^{n}, z_{c}^{n}\right\}$ and the summation in Eq. (2) runs over values which satisfy the relation $-1 \leq\left(\zeta_{g}^{n}-\zeta_{i}^{n}\right) / h \leq 1$. Note that $\mathbf{r}_{i}=\mathbf{i} x_{i}+\mathbf{j} y_{i}+\mathbf{k} z_{i}, x_{i}^{n}=x_{i}-x_{a}^{c}+\left(N_{x}-1\right) h / 2$, $y_{i}^{n}=y_{i}-y_{b}^{c}+\left(N_{y}-1\right) h / 2$, and $z_{i}^{n}=z_{i}-z_{c}^{c}+\left(N_{z}-1\right) h / 2$. The 3rd-order B-spline functions $M_{3}(\zeta)=\frac{\zeta}{2} M_{2}(\zeta)+\frac{3-\zeta}{2} M_{2}(\zeta-1)$ are continuous functions

$$
M_{3}(\zeta)=\left\{\begin{array}{rr}
\zeta^{2} / 2, & 0 \leq \zeta \leq 1 \\
-\zeta^{2}+3 \zeta-3 / 2, & 1 \leq \zeta<2 \\
(3-\zeta)^{2} / 2, & 2 \leq \zeta \leq 3
\end{array}\right.
$$

with $0 \leq \zeta \leq 3$. Thus, $\zeta$ is defined as $\zeta=3 / 2+\left(\zeta_{g}^{n}-\zeta_{i}^{n}\right) / h$ and the summation in Eq. (2) runs over values which satisfy the relation $-3 / 2 \leq\left(\zeta_{g}^{n}-\zeta_{i}^{n}\right) / h \leq 3 / 2$.

The electrostatic force acting on the $i$ particle due to the static potential is given by

$$
\mathbf{f}_{i}^{s f}=-q_{i} \boldsymbol{\nabla}_{i} \phi_{s f}^{m}\left(\mathbf{r}_{i}\right)
$$

Since $\phi_{s f}(a, b, c)$ is independent of the particle positions, the first derivative in Eq. (2) is given by

$$
\begin{aligned}
\nabla_{i} \phi_{s f}^{m}\left(\mathbf{r}_{i}\right) & =-h^{-1} \sum_{a, b, c}\left(\mathbf{i} \frac{d M_{m}(x)}{d x} M_{m}(y) M_{m}(z)+\mathbf{j} M_{m}(x) \frac{d M_{m}(y)}{d y} M_{m}(z)\right. \\
& \left.+\mathbf{k} M_{m}(x) M_{m}(y) \frac{d M_{m}(z)}{d z}\right) \phi_{s f}(a, b, c) .
\end{aligned}
$$

The first derivative of $M_{2}(\zeta)$ is given by

$$
\frac{d M_{2}(\zeta)}{d \zeta}=\left\{\begin{aligned}
1, & 0 \leq \zeta<1 \\
0, & \zeta=1 \\
-1, & 1<\zeta \leq 2
\end{aligned}\right.
$$


As we can observe, this function exhibits a discontinuity at $\zeta=1$. The first derivative of $M_{3}(\zeta)$ is given by

$$
\frac{d M_{3}(\zeta)}{d \zeta}=\left\{\begin{array}{rr}
\zeta, & 0 \leq \zeta \leq 1 \\
3-2 \zeta, & 1 \leq \zeta<2 \\
\zeta-3, & 2 \leq \zeta \leq 3
\end{array}\right.
$$

This derivative is given by a continuous function. It can be shown that

$$
\frac{d M_{3}(\zeta)}{d \zeta}=M_{2}(\zeta)-M_{2}(\zeta-1)
$$

The original core-repulsive potential is also calculated on a discrete grid and stored for computational efficiency. The entire simulation region is scanned at all grid points and a large positive energy is assigned to $\phi_{\text {core }}(a, b, c)$ if the distance to a fixed channel atom is less than the combined LJ radius (particle and fixed channel atom). It should be noted that the form of the core repulsion is isomorphic to that of an ion of charge unity interacting with a positive electrostatic potential. Therefore, the energy $U_{\text {core }}\left(\mathbf{r}_{i}\right)$ and its first derivatives are then calculated using the same method as described above.

\subsection{Reaction field potential}

\subsubsection{Generalized basis-set expansion}

A general method has been developed to include the electrostatic reaction field in BD simulations for particle dynamics across complex molecular channels of arbitrary geometry [3]. In this method, the PMF contribution from the reaction field is given by

$$
\Delta W_{r f}\left(\mathbf{r}_{1}, \ldots, \mathbf{r}_{N}\right)=\frac{1}{2} \sum_{m, n}^{M} Q_{m} M_{m n}^{*} Q_{n},
$$

where

$$
Q_{n}=\sum_{i}^{N} q_{i} b_{n}\left(\mathbf{r}_{i}\right)
$$

is the $n$ th-component of the generalized multipole moment $\mathbf{Q}$, and

$$
M_{m n}^{*}=\sum_{i, j}^{M} S_{i m}^{-1} M_{i j} S_{j n}^{-1}
$$

is a constant matrix for the system which represents the reaction field Green's function between the generated multipolar basis functions (multipoles). Note that $\left\{b_{m}(\mathbf{r})\right\}$ denotes a normalized basis set with $\mathrm{M}$ basis functions, the overlap matrix $\mathbf{S}$ is given by

$$
S_{n m}=\int d \mathbf{r} b_{n}(\mathbf{r}) b_{m}(\mathbf{r}),
$$

and

$$
M_{n m}=\int d \mathbf{r} b_{n}(\mathbf{r}) \phi_{r f}\left(\mathbf{r} ; b_{m}(\mathbf{r})\right),
$$

where $\phi_{r f}\left(\mathbf{r} ; b_{j}(\mathbf{r})\right)$ is the reaction field due to basis function $b_{m}(\mathbf{r})$. If a real basis set is used, $\mathbf{M}^{*}$ is a symmetric matrix. Real spherical harmonics and Legendre polynomials are used as basis 
set in all particle-accessible regions (see appendix in Ref. 4). The reaction field potential arising from the dielectric hetero-junction between solvent, protein, and lipid is defined as

$$
\phi_{r f}\left(\mathbf{r}_{i}\right) \equiv \frac{\partial \Delta W_{r f}\left(\mathbf{r}_{1}, \ldots, \mathbf{r}_{N}\right)}{\partial q_{i}}=\sum_{m, n}^{M} Q_{m} M_{m n}^{*} b_{n}\left(\mathbf{r}_{i}\right)
$$

and the PMF contribution from the reaction field can be also written as

$$
\Delta W_{r f}\left(\mathbf{r}_{1}, \ldots, \mathbf{r}_{N}\right)=\frac{1}{2} \sum_{i}^{N} q_{i} \phi_{r f}\left(\mathbf{r}_{i}\right) .
$$

The first derivative of Eq. (15) is given by

$$
\boldsymbol{\nabla}_{i} \phi_{r f}\left(\mathbf{r}_{i}\right)=\sum_{m, n}^{M}\left[q_{i} b_{m}\left(\mathbf{r}_{i}\right)+Q_{m}\right] M_{m n}^{*} \nabla_{i} b_{n}\left(\mathbf{r}_{i}\right) .
$$

Since $\phi_{r f}\left(\mathbf{r}_{i}\right)$ depends parametrically on the rest of particle positions, the cross term in the first derivative

$$
\nabla_{i} \phi_{r f}\left(\mathbf{r}_{j}\right)=q_{i} \sum_{m, n}^{M} \nabla_{i} b_{m}\left(\mathbf{r}_{i}\right) M_{m n}^{*} b_{n}\left(\mathbf{r}_{j}\right), j \neq i
$$

does not vanish. Thus, the force on particle $i$ due to the reaction field can be written as

$$
\mathbf{f}_{i}^{r f}=-\frac{1}{2}\left[q_{i} \boldsymbol{\nabla}_{i} \phi_{r f}\left(\mathbf{r}_{i}\right)+\sum_{j \neq i}^{N} q_{j} \boldsymbol{\nabla}_{i} \phi_{r f}\left(\mathbf{r}_{j}\right)\right]=-q_{i} \sum_{m, n}^{M} Q_{m} M_{m n}^{*} \boldsymbol{\nabla}_{i} b_{n}\left(\mathbf{r}_{i}\right) .
$$

Basis set in a orthorhombic inner region. Orthonormal basis functions for a variety of multipole charge distributions inside a orthorhombic simulation box can be defined as the products of the Legrende polynomials, $\left\{P_{l}\right\}$, in $x, y$ and $z$ directions,

$$
f_{l m n}(\mathbf{r})=\left[\frac{2 l+1}{L_{x}} \frac{2 m+1}{L_{y}} \frac{2 n+1}{L_{z}}\right]^{1 / 2} P_{l}\left(\frac{2}{L_{x}} x\right) P_{m}\left(\frac{2}{L_{y}} y\right) P_{n}\left(\frac{2}{L_{z}} z\right),
$$

where $L_{x}, L_{y}$ and $L_{z}$ are the lengths of the simulation box in each direction, and $\mathbf{r}=\mathbf{i} x+\mathbf{j} y+\mathbf{k} z$ with $x \in\left[-L_{x} / 2, L_{x} / 2\right], y \in\left[-L_{y} / 2, L_{y} / 2\right]$, and $z \in\left[-L_{z} / 2, L_{z} / 2\right]$. In Eq. (20), the cartesian coordinates are scaled because the Legrende polynomials are only defined between -1 and 1 . Note that the $l=0, \ldots, L, m=0, \ldots, M$ and $n=0, \ldots, N$, where $L, M$ and $N$ are the upper limits of the Legrende polynomials in each direction. The basis set functions $b_{l m n}(\mathbf{r})$ are then generated with a shape function $\Omega(\mathbf{r})$ which is equal to one in all ion-accesible regions and zero otherwise,

$$
b_{l m n}(\mathbf{r})=\Omega(\mathbf{r}) f_{l m n}(\mathbf{r}) .
$$

The Legrende polynomials can be obtained from the recurrence relation

$$
(n+1) P_{n+1}(x)=(2 n+1) x P_{n}(x)-n P_{n-1}(x),
$$

where the initial values are $P_{0}(x)=1$ and $P_{1}(x)=x$. The first derivate of the Legrende polynomials can be obtained from the recurrence relation

$$
\frac{d P_{n+1}(x)}{d x}=(n+1) P_{n}(x)+x \frac{d P_{n}(x)}{d x},
$$

where the initial value is $\frac{d P_{0}(x)}{d x}=0$. 
Basis set in a spherical inner region. The orthonormal basis functions of the $l$ th multipole charge distribution inside a spherical simulation box of radius $R$ are defined as

$$
f_{l m}(\mathbf{r})=N_{l m} r^{l} S_{l m}(\theta, \phi)
$$

where

$$
\begin{gathered}
N_{l m}=\sqrt{\frac{2 l+1}{2 \pi\left(1+\delta_{m, 0}\right)} \frac{2 l+3}{R^{2 l+3}} \frac{(l-|m|) !}{(l+|m|) !}}, \\
S_{l m}(\theta, \phi)=P_{l}^{|m|}(\cos \theta) \Phi_{m}(\phi),
\end{gathered}
$$

and

$$
\Phi_{m}(\phi)= \begin{cases}\cos m \phi & m>0 \\ 1 & m=0 \\ \sin |m| \phi & m<0\end{cases}
$$

Note that $l=0, \ldots, L$ and $m=-l, \ldots, 0, \ldots, l$ are the angular and azimuthal quantum numbers, respectively. The polar spherical coordinates $(r, \theta, \phi)$ are given by

$$
\begin{array}{rll}
r^{2}=x^{2}+y^{2}+z^{2} & , & 0 \leq r \leq \infty \\
\cos \theta=\frac{z}{r} & , & 0 \leq \theta \leq \pi \\
\tan \phi=\frac{y}{x} & , & 0 \leq \phi \leq 2 \pi
\end{array}
$$

In Eq. (26), $P_{l}^{m}(x)$ are the associated Legendre polynomials. The basis set functions $b_{l m}(\mathbf{r})$ are given by

$$
b_{l m}(\mathbf{r})=\Omega(\mathbf{r}) f_{l m}(\mathbf{r}) .
$$

To obtain the $S_{l m}(\theta, \phi)$ functions, one can use closed formulas for the associated Legendre polynomials, and then evaluate directly $\cos m \phi$ and $\sin m \phi$. However, this can lead to numerical instabilities, in addition to being inefficient when, as is usually the case, all the $S_{l m}$ up to a given $l$ are needed. In this case, it is more efficient to use stable recurrence relations, such as

$$
\begin{aligned}
P_{l}^{l}(\cos \theta) & =(2 l-1) \sin \theta P_{l-1}^{l-1}(\cos \theta) \\
P_{l}^{l-1}(\cos \theta) & =(2 l-1) \cos \theta P_{l-1}^{l-1}(\cos \theta) \\
P_{l}^{m}(\cos \theta) & =\frac{(2 l-1) \cos \theta P_{l-1}^{m}(\cos \theta)-(l+m-1) P_{l-2}^{m}(\cos \theta)}{l-m}, m=0, \ldots, l-2 .
\end{aligned}
$$

The initial values for these recurrence relations are $P_{0}^{0}(\cos \theta)=1, P_{1}^{1}(\cos \theta)=\sin \theta$, and $P_{1}^{0}(\cos \theta)=\cos \theta$. For $\cos m \phi$ and $\sin m \phi$ functions, one can start with the values $\sin \phi=$ $y /(r \sin \theta)$ and $\cos \phi=x /(r \sin \theta)$, and then use the known trigonometric formulas:

$$
\begin{aligned}
\cos m \phi & =\sin \phi \cos (m-1) \phi+\cos \phi \sin (m-1) \phi \\
\sin m \phi & =\cos \phi \cos (m-1) \phi-\sin \phi \sin (m-1) \phi .
\end{aligned}
$$

Using the above recurrence relations, one can obtain the Legrende and trigonometric functions separately, so that no one expression is computed more than once, because only Legendre polynomials with $m \geq 0$ are needed, and trigonometric functions for a given $m$ are common for all $l$ values. The normalization factors $N_{l m}$ need to be computed only once at the beginning 
of a simulation. To evaluate $\nabla f_{l m}(\mathbf{r})$, one should use the relations:

$$
\begin{aligned}
\boldsymbol{\nabla} & =\mathbf{i} \frac{\partial}{\partial x}+\mathbf{j} \frac{\partial}{\partial y}+\mathbf{k} \frac{\partial}{\partial z}, \\
\frac{\partial}{\partial x} & =\sin \theta \cos \phi \frac{\partial}{\partial r}+\frac{\cos \theta \cos \phi}{r} \frac{\partial}{\partial \theta}-\frac{\sin \phi}{r \sin \theta} \frac{\partial}{\partial \phi}, \\
\frac{\partial}{\partial y} & =\sin \theta \sin \phi \frac{\partial}{\partial r}+\frac{\cos \theta \sin \phi}{r} \frac{\partial}{\partial \theta}+\frac{\cos \phi}{r \sin \theta} \frac{\partial}{\partial \phi}, \\
\frac{\partial}{\partial z} & =\cos \theta \frac{\partial}{\partial r}-\frac{\sin \theta}{r} \frac{\partial}{\partial \theta} .
\end{aligned}
$$

Thereby, the explicit expressions for $\nabla f_{l m}(\mathbf{r})$ are given by

$$
\begin{aligned}
\frac{\partial f_{l m}(\mathbf{r})}{\partial x} & =N_{l m} r^{l-1}\left[l \sin \theta \cos \phi S_{l m}(\theta, \phi)+\cos \theta \cos \phi \frac{\partial S_{l m}(\theta, \phi)}{\partial \theta}-\frac{\sin \phi}{\sin \theta} \frac{\partial S_{l m}(\theta, \phi)}{\partial \phi}\right] \\
\frac{\partial f_{l m}(\mathbf{r})}{\partial y} & =N_{l m} r^{l-1}\left[l \sin \theta \sin \phi S_{l m}(\theta, \phi)+\cos \theta \sin \phi \frac{\partial S_{l m}(\theta, \phi)}{\partial \theta}+\frac{\cos \phi}{\sin \theta} \frac{\partial S_{l m}(\theta, \phi)}{\partial \phi}\right] \\
\frac{\partial f_{l m}(\mathbf{r})}{\partial z} & =N_{l m} r^{l-1}\left[l \cos \theta S_{l m}(\theta, \phi)-\sin \theta \frac{\partial S_{l m}(\theta, \phi)}{\partial \theta}\right]
\end{aligned}
$$

where

$$
\begin{aligned}
\frac{\partial S_{l m}(\theta, \phi)}{\partial \theta} & =\Phi_{m}(\phi) \frac{d P_{l}^{|m|}(\cos \theta)}{d \theta} \\
\frac{\partial S_{l m}(\theta, \phi)}{\partial \phi} & =P_{l}^{|m|}(\cos \theta) \frac{d \Phi_{m}(\phi)}{d \phi} \\
\frac{d \Phi_{m}(\phi)}{d \phi} & = \begin{cases}-m \sin m \phi & m>0, \\
0 & m=0 \\
|m| \cos |m| \phi & m<0\end{cases}
\end{aligned}
$$

To calculate the first derivative of the associated Legendre polynomials, one can use the recurrence relation:

$$
\frac{d P_{l}^{m}(\cos \theta)}{d \theta}=m \frac{\cos \theta}{\sin \theta} P_{l}^{m}(\cos \theta)-P_{l}^{m+1}(\cos \theta) .
$$

Notice that $P_{l}^{m}(x)=0$ if $m>l$.

In Eqs. (39), (40) and (45) some singularities appears when the cartesian position is along the $z$ axis, i.e., $\sin \theta=0$. To avoid this problem in practical applications, the $\theta$ value can be restricted to $(0, \pi)$.

\subsubsection{Generalized Born model}

The PMF contribution from the reaction field is given by

$$
\Delta W_{r f}\left(\mathbf{r}_{1}, \ldots, \mathbf{r}_{N}\right)=\frac{1}{2} \sum_{i}^{N} q_{i} \phi_{r f}\left(\mathbf{r}_{i}\right)=\frac{1}{2} \sum_{i}^{N} q_{i} G_{R F}\left(\mathbf{r}_{i}, \mathbf{r}_{i}\right) q_{i}+\sum_{i>j}^{N} q_{i} G_{R F}\left(\mathbf{r}_{i}, \mathbf{r}_{j}\right) q_{j},
$$

where $\phi_{r f}\left(\mathbf{r}_{i}\right)$ is the reaction field at $\mathbf{r}_{i}$ and $G_{R F}\left(\mathbf{r}_{i}, \mathbf{r}_{j}\right)$ is the Green's function corresponding to the reaction field potential at $\mathbf{r}_{i}$ arising from a point charge located at $\mathbf{r}_{j}$. The reaction field is defined as $\phi_{r f}(\mathbf{r})=\phi_{S}(\mathbf{r})-\phi_{\text {bulk }}(\mathbf{r})$ where $\phi_{S}(\mathbf{r})$ is the electrostatic potential computed for the 
complex solvent-channel environment, and $\phi_{b u l k}(\mathbf{r})$ is the electrostatic potential computed for a reference system corresponding to a uniform bulk solvent at zero salt concentration.

A Generalized Born type model can be used for describing the PMF contribution from the reaction field. In this model, Eq. (46) is approximated by an analytical formula:

$$
\Delta W_{r f}\left(\mathbf{r}_{1}, \ldots, \mathbf{r}_{N}\right) \approx \Delta W_{r f}^{G B}\left(\mathbf{r}_{1}, \ldots, \mathbf{r}_{N}\right)=\Delta W_{r f}^{s t}\left(\mathbf{r}_{1}, \ldots, \mathbf{r}_{N}\right)+\Delta W_{r f}^{c t}\left(\mathbf{r}_{1}, \ldots, \mathbf{r}_{N}\right),
$$

where the direct term is given by

$$
\Delta W_{r f}^{s t}\left(\mathbf{r}_{1}, \ldots, \mathbf{r}_{N}\right)=\frac{1}{2} \sum_{i}^{N}\left[q_{i} F\left(\mathbf{r}_{i}\right)\right]^{2}
$$

and the cross term by

$$
\Delta W_{r f}^{c t}\left(\mathbf{r}_{1}, \ldots, \mathbf{r}_{N}\right)=\sum_{i>j}^{N} q_{i} F\left(\mathbf{r}_{i}\right) q_{j} F\left(\mathbf{r}_{j}\right) \frac{R_{i}^{1 / 2} R_{j}^{1 / 2}}{\left(r_{i j}^{2}+R_{i} R_{j}\right)^{1 / 2}}
$$

In the previous expressions, one uses the function $F\left(\mathbf{r}_{i}\right)=\left[G_{R F}\left(\mathbf{r}_{i}, \mathbf{r}_{i}\right)\right]^{1 / 2}$ and the set $\left\{R_{i}\right\}_{i=1, \ldots, N}$ is composed by the effective Born radii. It should be stressed that $G_{R F}\left(\mathbf{r}_{i}, \mathbf{r}_{i}\right) \equiv$ $-\tau / R_{i}$ where $\tau=1 / \varepsilon_{\text {bulk }}-1 / \varepsilon_{p}$ being $\varepsilon_{\text {bulk }}$ and $\varepsilon_{p}$ the dielectric response in water and nonallowed particle regions, respectively.

The force on particle $i$ due to the reaction field is

$$
\mathbf{f}_{i}^{r f}=\mathbf{f}_{i}^{r f, s t}+\frac{1}{2} \sum_{j \neq i} \mathbf{f}_{i j}^{r f, c t}
$$

where

$$
\begin{gathered}
\mathbf{f}_{i}^{r f, s t}=-q_{i}^{2} F\left(\mathbf{r}_{i}\right) \boldsymbol{\nabla}_{i} F\left(\mathbf{r}_{i}\right), \\
\mathbf{f}_{i j}^{r f, c t}=\mathbf{f}_{i j}^{r f, c t, I}+\mathbf{f}_{i j}^{r f, c t, I I}+\mathbf{f}_{i j}^{r f, c t, I I I}, \\
\mathbf{f}_{i j}^{r f, c t, I}=-q_{i} q_{j} F\left(\mathbf{r}_{j}\right) \frac{R_{i}^{1 / 2} R_{j}^{1 / 2}}{\left(r_{i j}^{2}+R_{i} R_{j}\right)^{1 / 2}} \nabla_{i} F\left(\mathbf{r}_{i}\right), \\
\mathbf{f}_{i j}^{r f, c t, I I}=-q_{i} q_{j} F\left(\mathbf{r}_{i}\right) F\left(\mathbf{r}_{j}\right) \frac{R_{i}^{1 / 2} R_{j}^{1 / 2}}{\left(r_{i j}^{2}+R_{i} R_{j}\right)^{3 / 2}} \mathbf{r}_{i j}, \\
\mathbf{f}_{i j}^{r f, c t, I I I}=-q_{i} q_{j} F\left(\mathbf{r}_{i}\right) F\left(\mathbf{r}_{j}\right) \frac{R_{j}^{1 / 2} r_{i j}^{2}}{\left(r_{i j}^{2}+R_{i} R_{j}\right)^{3 / 2}} \nabla_{i} R_{i}^{1 / 2} .
\end{gathered}
$$
by

In the discrete grid approximation, the functions $F\left(\mathbf{r}_{i}\right)$ and the effective Born radii are given

$$
\begin{aligned}
F\left(\mathbf{r}_{i}\right) & =\sum_{a, b, c} M_{m}(x) M_{m}(y) M_{m}(z) F(a, b, c), \\
R_{i}^{1 / 2} & =\sum_{a, b, c} M_{m}(x) M_{m}(y) M_{m}(z)[R(a, b, c)]^{1 / 2},
\end{aligned}
$$

where $F(a, b, c)$ and $R(a, b, c)$ are the values of the function $F$ and effective Born radius at the grid point $\left(x_{a}^{n}, y_{b}^{n}, z_{c}^{n}\right)$ (see Eq. (1)), and $\left\{M_{m}\right\}_{m=2,3}$ are the 2nd-order and 3rd-order B-spline 
functions. The gradients from Eqs. (56) and (57) are given by

$$
\begin{aligned}
\boldsymbol{\nabla}_{i} F\left(\mathbf{r}_{i}\right) & =\sum_{a, b, c}\left(\mathbf{i} \frac{\partial M_{m}(x)}{\partial x_{i}} M_{m}(y) M_{m}(z)+\mathbf{j} M_{m}(x) \frac{\partial M_{m}(y)}{\partial y_{i}} M_{m}(z)\right. \\
& \left.+\mathbf{k} M_{m}(x) M_{m}(y) \frac{\partial M_{m}(z)}{\partial z_{i}}\right) F(a, b, c) \\
\boldsymbol{\nabla}_{i} R_{i}^{1 / 2} & =\sum_{a, b, c}\left(\mathbf{i} \frac{\partial M_{m}(x)}{\partial x_{i}} M_{m}(y) M_{m}(z)+\mathbf{j} M_{m}(x) \frac{\partial M_{m}(y)}{\partial y_{i}} M_{m}(z)\right. \\
& \left.+\mathbf{k} M_{m}(x) M_{m}(y) \frac{\partial M_{m}(z)}{\partial z_{i}}\right)[R(a, b, c)]^{1 / 2}
\end{aligned}
$$

\section{SHAKE algorithm}

In the improved GCMC/BD algorithm, bond-length constraints can be applied on explicit atoms for the reasons discussed in the main text (see Section 3.6). In this section, we describe a version of the SHAKE [5] algorithm for using in the BD framework which has been implemented in the BROMOCEA code.

The $N_{c}$ distance constraints within a specific molecule can be written in the form of $N_{c}$ constraint equations

$$
\sigma_{k}\left(\mathbf{r}_{1}, \ldots, \mathbf{r}_{N}\right)=r_{k_{1} k_{2}}^{2}-d_{k}^{2}=0, k=1, \ldots, N_{c}
$$

where $\mathbf{r}_{k_{1} k_{2}}=\mathbf{r}_{k_{2}}-\mathbf{r}_{k_{1}}, r_{k_{1} k_{2}}$ is the distance between atoms $k_{1}$ and $k_{2}$ involved in constraint $k$, and $d_{k}$ is the corresponding constraint distance. If the EM algorithm is used to generate a $\mathrm{BD}$ trajectory, the unconstrained atomic coordinates resulting from the action of the unconstrained forces are given by

$$
\mathbf{r}_{i}^{u c}(t+\Delta t)=\mathbf{r}_{i}(t)+\nabla_{i} D_{i}(t) \Delta t+\frac{D_{i}(t) \Delta t}{k_{B} T} \mathbf{F}_{i}^{u c}(t)+\mathbf{R}_{i}(\Delta t)
$$

where

$$
\mathbf{F}_{i}^{u c}(t)=-\nabla_{i} W\left(\mathbf{r}_{1}(t), \ldots, \mathbf{r}_{N}(t)\right)
$$

and $\mathbf{R}_{i}(\Delta t)$ is a random displacement with a gaussian distribution which obeys

$$
\begin{aligned}
\left\langle\mathbf{R}_{i}(\Delta t)\right\rangle & =\mathbf{0} \\
\left\langle\mathbf{R}_{i}(\Delta t) \cdot \mathbf{R}_{i}(\Delta t)\right\rangle & =6 D_{i}(t) \Delta t .
\end{aligned}
$$

Adding the effect of the constraint forces leads to

$$
\mathbf{r}_{i}(t+\Delta t)=\mathbf{r}_{i}^{u c}(t+\Delta t)+\frac{D_{i}(t) \Delta t}{k_{B} T} \mathbf{F}_{i}^{c}(t),
$$

where

$$
\mathbf{F}_{i}^{c}(t)=-2 \sum_{k=1}^{N_{c}} \lambda_{k}(t)\left(\delta_{i, k_{2}}-\delta_{i, k_{1}}\right) \mathbf{r}_{k_{1} k_{2}}(t),
$$

and $\lambda_{k}$ are the (unknown) Lagrange multipliers. It should be stressed that the SHAKE algorithm also works properly in combination with the SRK algorithm since it only modifies the determination of the unconstrained coordinates, Eq. (61), but does not alter the application 
of the holonomic constraints described below. The $\mathbf{r}_{i}(t+\Delta t)$ coordinates should satisfy the constraint equations, and thus

$$
\left[\mathbf{r}_{k_{1} k_{2}}^{u c}(t+\Delta t)+\frac{D_{k_{2}}(t) \Delta t}{k_{B} T} \mathbf{F}_{k_{2}}^{c}(t)-\frac{D_{k_{1}}(t) \Delta t}{k_{B} T} \mathbf{F}_{k_{1}}^{c}(t)\right]^{2}-d_{k}^{2}=0, k=1, \ldots, N_{c} .
$$

Inserting Eq. (66) leads to a set of $N_{c}$ quadratic equations, which is to be solved for the Lagrange multipliers $\lambda_{k}$.

In the SHAKE method [5], the following two approximations are made: (1) the system of equations is linearized by neglecting the terms quadratic in the Lagrange multipliers, and (2) the equations are decoupled by assuming that the atoms $k_{1}$ and $k_{2}$ involved in constraint $k$ are not involved in any other constraint. With these simplifications, approximate solutions for the multipliers are given by

$$
\lambda_{k}(t)=\frac{k_{B} T}{4 \Delta t} \frac{\left[\mathbf{r}_{k_{1} k_{2}}^{u c}(t+\Delta t)\right]^{2}-d_{k}^{2}}{\left[D_{k_{1}}(t)+D_{k_{2}}(t)\right] \mathbf{r}_{k_{1} k_{2}}^{u c}(t+\Delta t) \cdot \mathbf{r}_{k_{1} k_{2}}(t)},
$$

and the corresponding coordinate adjustments are performed according to Eqs. (65) and (66) as

$$
\begin{aligned}
& \mathbf{r}_{k_{1}}(t+\Delta t)=\mathbf{r}_{k_{1}}^{u c}(t+\Delta t)+2 \frac{D_{k_{1}}(t) \Delta t}{k_{B} T} \lambda_{k}(t) \mathbf{r}_{k_{1} k_{2}}(t), \\
& \mathbf{r}_{k_{2}}(t+\Delta t)=\mathbf{r}_{k_{2}}^{u c}(t+\Delta t)-2 \frac{D_{k_{2}}(t) \Delta t}{k_{B} T} \lambda_{k}(t) \mathbf{r}_{k_{1} k_{2}}(t) .
\end{aligned}
$$

Inserting Eq. (68) into Eqs. (69) and (70), one obtains

$$
\begin{aligned}
& \mathbf{r}_{k_{1}}(t+\Delta t)=\mathbf{r}_{k_{1}}^{u c}(t+\Delta t)+\frac{D_{k_{1}}(t)\left\{\left[\mathbf{r}_{k_{1} k_{2}}^{u c}(t+\Delta t)\right]^{2}-d_{k}^{2}\right\}}{2\left[D_{k_{1}}(t)+D_{k_{2}}(t)\right] \mathbf{r}_{k_{1} k_{2}}^{u c}(t+\Delta t) \cdot \mathbf{r}_{k_{1} k_{2}}(t)} \mathbf{r}_{k_{1} k_{2}}(t), \\
& \mathbf{r}_{k_{2}}(t+\Delta t)=\mathbf{r}_{k_{2}}^{u c}(t+\Delta t)-\frac{D_{k_{2}}(t)\left\{\left[\mathbf{r}_{k_{1} k_{2}}^{u c}(t+\Delta t)\right]^{2}-d_{k}^{2}\right\}}{2\left[D_{k_{1}}(t)+D_{k_{2}}(t)\right] \mathbf{r}_{k_{1} k_{2}}^{u c}(t+\Delta t) \cdot \mathbf{r}_{k_{1} k_{2}}(t)} \mathbf{r}_{k_{1} k_{2}}(t) .
\end{aligned}
$$

Due to the approximations made above, the procedure must be iterated, taking the coordinates $\mathbf{r}_{i}$ obtained through Eqs. (71) and (72) at each iteration as the coordinates $\mathbf{r}_{i}^{u c}$ for the next iteration. The iteration cycle for a given molecule is terminated when all constraint conditions, Eq. (60), are satisfied within a given tolerance

$$
\frac{\left|r_{k_{1} k_{2}}^{2}(t+\Delta t)-d_{k_{1} k_{2}}^{2}\right|}{2 d_{k_{1} k_{2}}^{2}} \leq \tau,
$$

where $\tau$ is a relative geometric tolerance on the constrained length.

\section{Random number generator}

The sampling in GCMC/BD simulations of stochastic processes relies on the generation of pseudorandom numbers given an initial seed. Different from the underlying BROMOC code, the MRG32k3a uniform random number generator [6] has been included in the BROMOCEA code. This algorithm is based on combining parallel multiple recursive sequences and, thus, it exhibits a long period length. MRG32k3a performed very well in several tests [7] and is widely used in simulations and statistical softwares. There are several techniques to set a variate from a normal distribution on the basis of uniformly distributed variate. An efficient approach [8] has been implemented in the BROMOCEA code. 
Table S1: Average number of ions in the channel $N$, average ratio $N_{K^{+}} / N_{C l^{-}}$and average conductance $G$ for original GCMC/BD simulations. The time step was set to $\Delta t=10$ fs. The switching distances $r_{\text {on }}$ and $r_{\text {off }}$ were set to 8 and $12 \AA$, respectively. Furthermore, the uncertainties were calculated using $\sigma / \sqrt{n}$ where $\sigma$ denotes the standard deviation and $n=10$ the number of simulations.

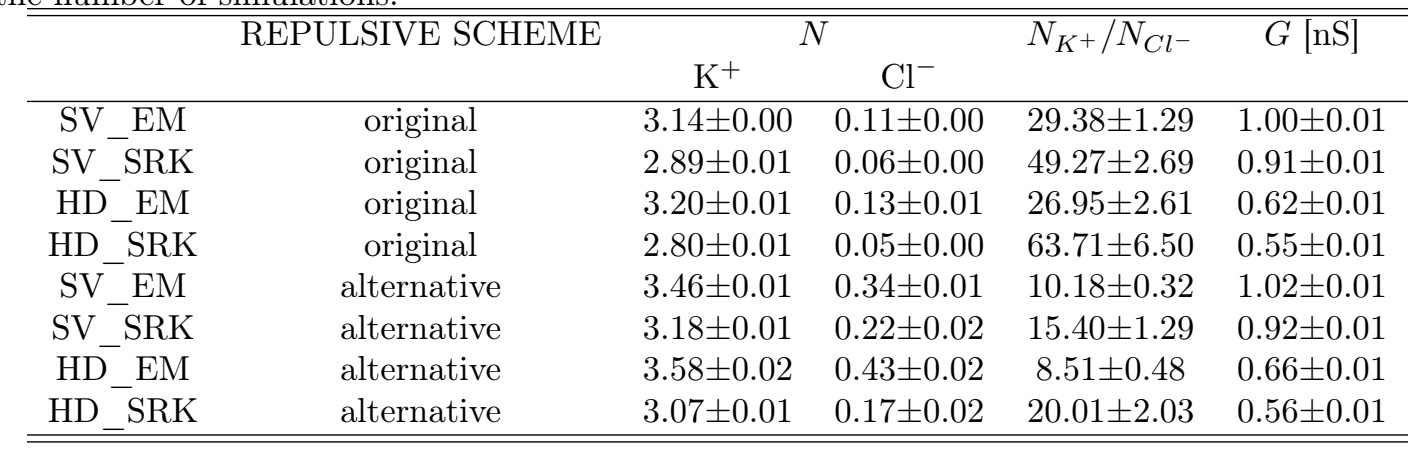

Table S2: Average number of ions in the channel $N$, average ratio $N_{K^{+}} / N_{C l^{-}}$and average conductance $G$ for restrained GCMC/BD simulations. The time step was set to $\Delta t=10 \mathrm{fs}$.

\begin{tabular}{cccccc}
\hline \hline & REPULSIVE SCHEME & \multicolumn{2}{c}{$\mathrm{N}$} & $N_{K^{+}} / N_{C l^{-}}$ & $\mathrm{G}[\mathrm{nS}]$ \\
& & $\mathrm{K}^{+}$ & $\mathrm{Cl}^{-}$ & & \\
\hline SV_EM & original & $7.89 \pm 0.12$ & $1.14 \pm 0.14$ & $5.77 \pm 0.45$ & $1.31 \pm 0.04$ \\
$\mathrm{SV}$ _SRK & original & $7.50 \pm 0.13$ & $0.93 \pm 0.15$ & $7.85 \pm 0.48$ & $1.30 \pm 0.05$ \\
$\mathrm{HD}$ _EM & original & $7.88 \pm 0.14$ & $1.02 \pm 0.17$ & $5.50 \pm 0.43$ & $0.79 \pm 0.02$ \\
SV_EM & alternative & $8.45 \pm 0.10$ & $1.57 \pm 0.14$ & $9.26 \pm 2.02$ & $1.42 \pm 0.04$ \\
SV_SRK & alternative & $7.67 \pm 0.05$ & $1.02 \pm 0.06$ & $7.42 \pm 1.59$ & $1.28 \pm 0.03$ \\
HD_EM & alternative & $8.46 \pm 0.13$ & $1.64 \pm 0.13$ & $10.73 \pm 2.06$ & $0.86 \pm 0.03$ \\
\hline \hline
\end{tabular}

\section{Additional results from GCMC/BD simulations}

Table S1 shows the results for the conductance and the average number of ions inside the channel for original GCMC/BD simulations where the switching distances $r_{\text {on }}$ and $r_{\text {off }}$ were set to 8 and $12 \AA$, respectively. Furthermore, the corresponding PMF profiles are displayed in Figs. S1 and S2. The conditions of the original GCMC/BD simulations are described in the main text (Section 5.4). Table S2 shows results for the conductance and the average number of ions inside the channel in restrained GCMC/BD simulations (see Section 5.5 of the main text).

Additional PMF profiles are displayed in Figs. S3 and S4. Table S3 gives additional results for the conductance and the average number of ions inside the channel in flexible GCMC/BD simulations (see Section 5.6 of the main text). The corresponding PMF profiles are displayed in Fig. S5.

By definition, the equilibrium condition in the ion translocation across a channel implies that the total average current is zero. Thus, the estimate of the total average current from simulations under equilibrium conditions can be viewed as an estimate of the stochastic component (or noise) in the current measurement. Tables S4 and S5 show the total average current for equilibrium simulations described in Section 5.4 of the main text where the switching distances $r_{o n}$ and $r_{o f f}$ were set to 15 and $20 \AA$, respectively. The total average currents for equilibrium simulations described in Section 5.4 of main text where the switching distances $r_{o n}$ and $r_{o f f}$ were set to 8 
a) SV model, EM propagator

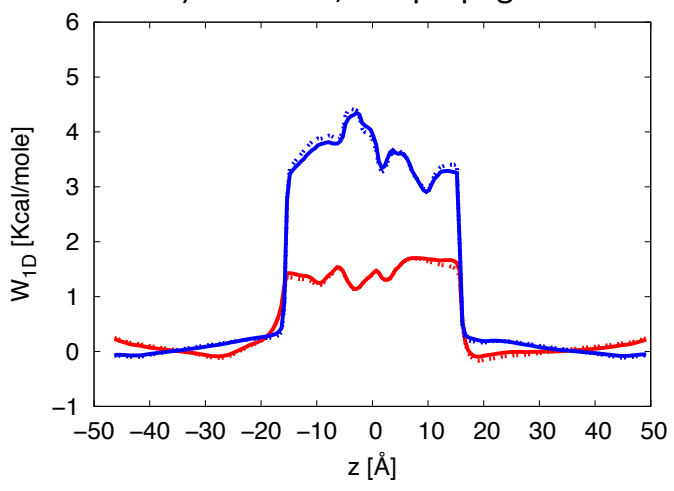

c) HD model, EM propagator

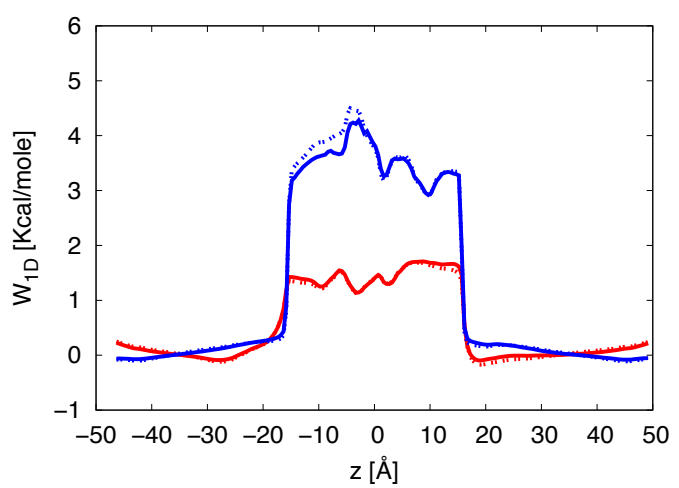

b) SV model, SRK propagator

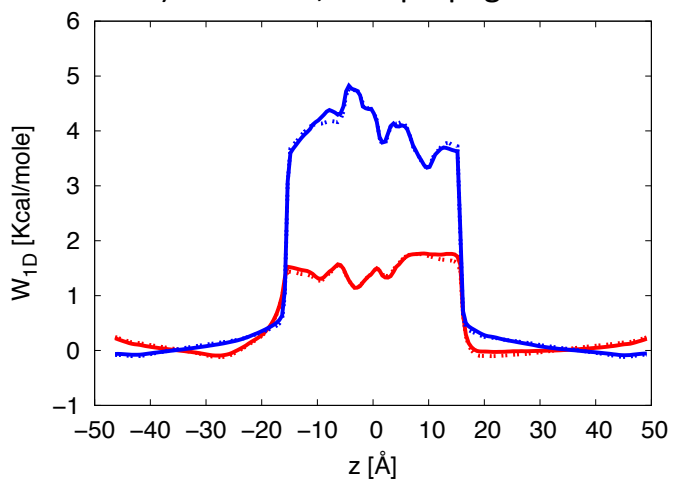

d) HD model, SRK propagator

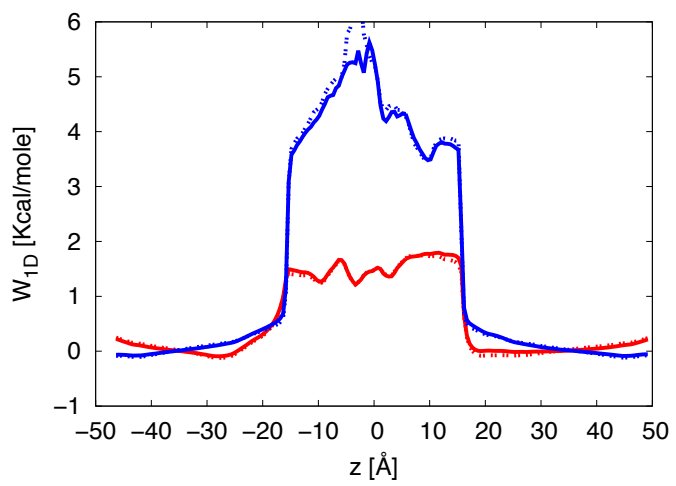

Figure S1: 1D multi-ion average PMF extracted from 10 independent original GMC/BD simulations under equilibrium conditions $\left(V_{m p}=0\right)$ using the original core-repulsive scheme. The results for the $\mathrm{K}^{+}$ions are represented in red while for the $\mathrm{Cl}^{-}$ions in blue. The time step was set to $\Delta t=10 \mathrm{fs}$. The different cutoff values $r_{o n}=15 \AA, r_{o f f}=20 \AA$ and $r_{o n}=8 \AA$, $r_{o f f}=12 \AA$ in the atom-based force switching method are represented in solid and dotted lines, respectively.

Table S3: Average number of ions in the channel $N$, average ratio $N_{K^{+}} / N_{C l^{-}}$and average conductance $G$ for flexible GCMC/BD simulations using the alternative core-repulsive potential scheme.

\begin{tabular}{cccccc}
\hline \hline & $\Delta t[\mathrm{fs}]$ & \multicolumn{2}{c}{$N$} & $N_{K^{+}} / N_{C l^{-}}$ & $G[\mathrm{nS}]$ \\
& & $\mathrm{K}^{+}$ & $\mathrm{Cl}^{-}$ & & \\
\hline SV_EM & 10 & $7.53 \pm 0.13$ & $1.29 \pm 0.13$ & $7.41 \pm 1.65$ & $1.09 \pm 0.11$ \\
SV_EM & 5 & $7.52 \pm 0.14$ & $1.20 \pm 0.16$ & $7.83 \pm 1.28$ & $1.15 \pm 0.11$ \\
SV_SRK & 10 & $6.94 \pm 0.06$ & $1.06 \pm 0.05$ & $6.72 \pm 0.33$ & $0.92 \pm 0.08$ \\
SV_SRK & 5 & $6.92 \pm 0.13$ & $1.00 \pm 0.17$ & $10.70 \pm 2.6$ & $0.95 \pm 0.07$ \\
HD_EM & 10 & $7.68 \pm 0.06$ & $1.33 \pm 0.06$ & $5.89 \pm 0.28$ & $0.73 \pm 0.07$ \\
HD_EM & 5 & $7.57 \pm 0.12$ & $1.29 \pm 0.16$ & $14.53 \pm 8.48$ & $0.70 \pm 0.08$ \\
\hline \hline
\end{tabular}


a) SV model, EM propagator

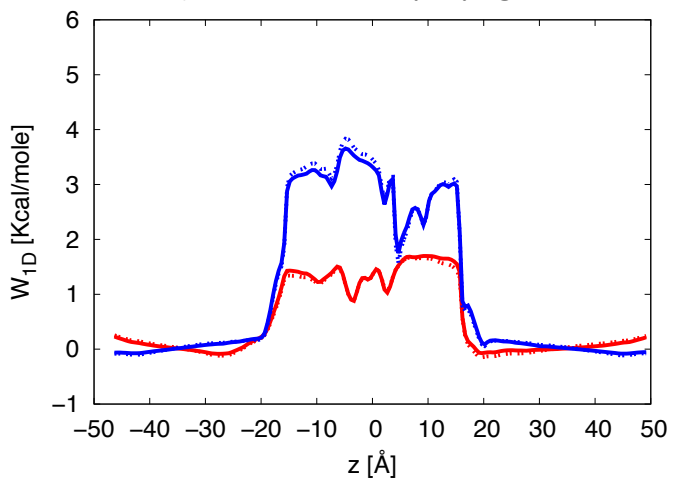

c) HD model, EM propagator

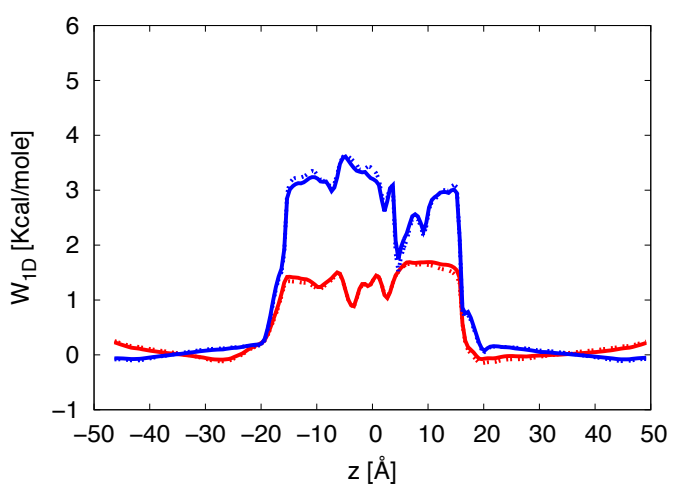

b) SV model, SRK propagator

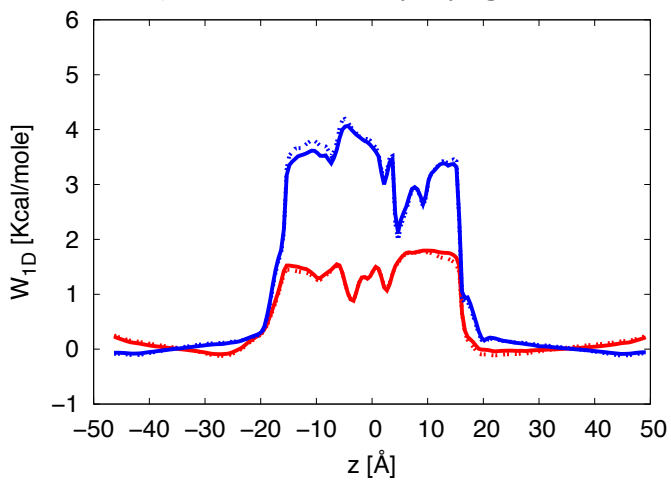

d) HD model, SRK propagator

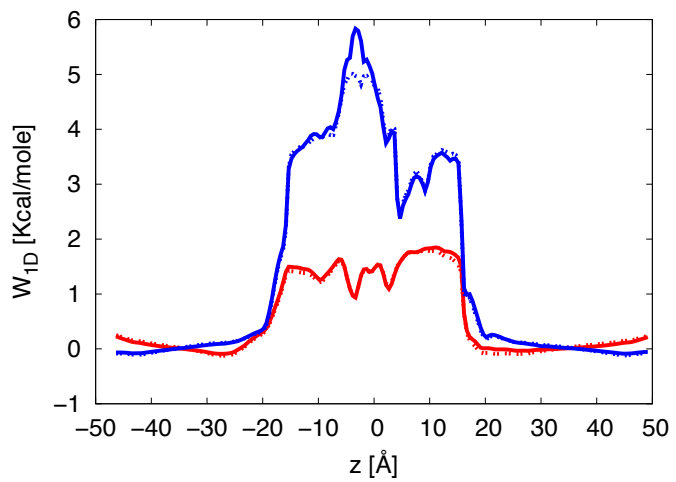

Figure S2: Same as in Figure S1 but using the alternative core-repulsive scheme.

Table S4: Total average current $(I)$ for original GCMC/BD simulations at equilibrium conditions using the original core-repulsive potential scheme. The switching distances $r_{\text {on }}$ and $r_{\text {off } f}$ were set to 15 and $20 \AA$, respectively.

\begin{tabular}{ccc}
\hline \hline & $\Delta t[\mathrm{fs}]$ & $I[\mathrm{pA}]$ \\
\hline SV_EM & 10 & $-1 \pm 1$ \\
SV_SRK & 10 & $-3 \pm 4$ \\
HD_EM & 10 & $-1 \pm 1$ \\
HD_SRK & 10 & $-2 \pm 1$ \\
SV_EM & 50 & $-2 \pm 1$ \\
SV_SRK & 50 & $-2 \pm 1$ \\
HD_EM & 50 & $-3 \pm 0$ \\
HD_SRK & 50 & $-2 \pm 0$ \\
SV_EM & 100 & $-4 \pm 1$ \\
SV_SRK & 100 & $-1 \pm 1$ \\
HD_EM & 100 & $-1 \pm 1$ \\
HD_SRK & 100 & $-1 \pm 1$ \\
\hline \hline
\end{tabular}


a) SV model, EM propagator

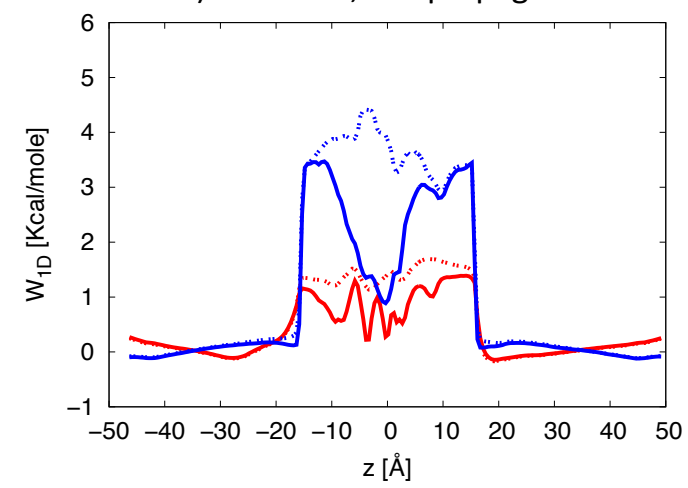

b) SV model, SRK propagator
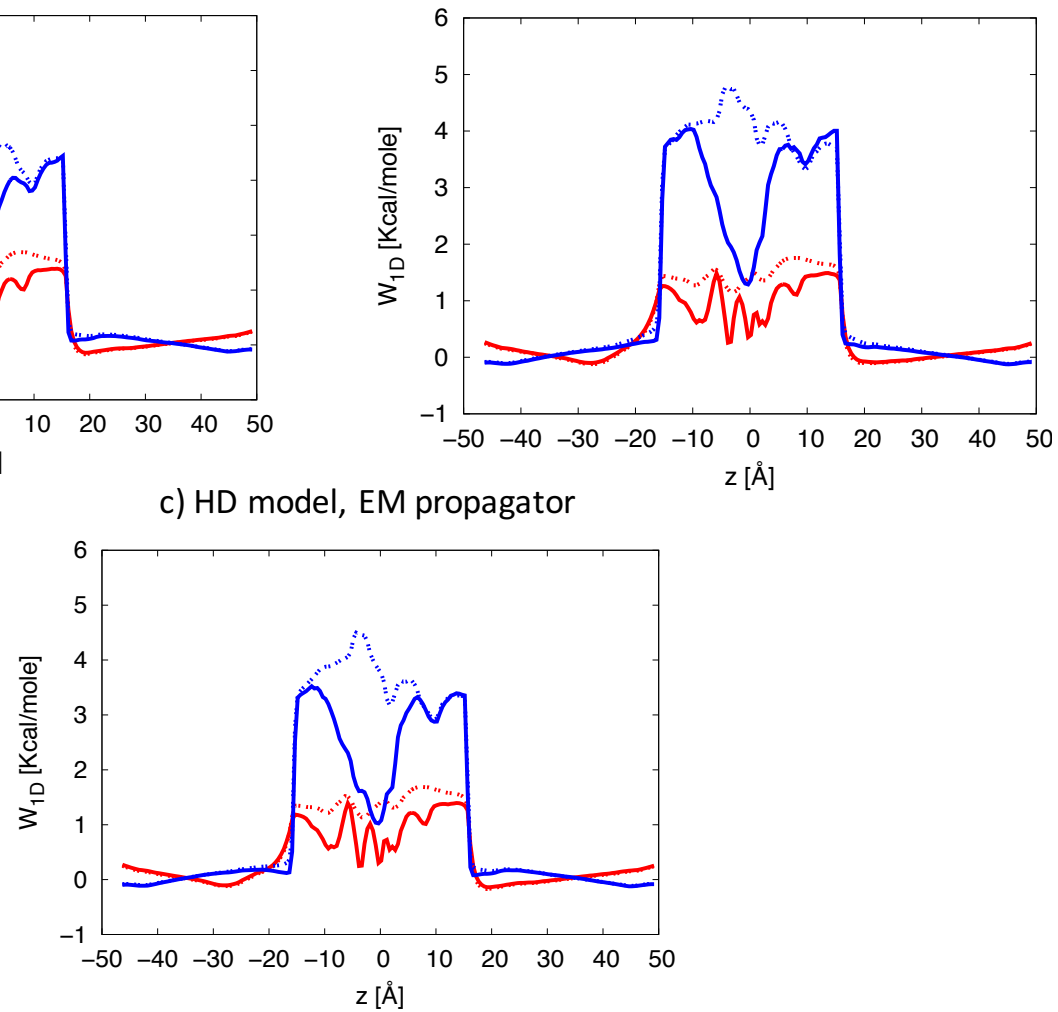

Figure S3: 1D multi-ion average PMF extracted from 10 independent restrained GMC/BD simulations under equilibrium conditions $\left(V_{m p}=0\right)$ using the original core-repulsive scheme. The results for the $K^{+}$ions are represented in red while for the $\mathrm{Cl}^{-}$ions in blue. To facilite comparison, the multi-ion PMF for the respective original GCMC/BD simulations (see Figure S1) are given as dotted lines.

Table S5: Same as in Table S4 but using the alternative core-repulsive potential scheme.

\begin{tabular}{ccc}
\hline \hline & $\Delta t[\mathrm{fs}]$ & $I[\mathrm{pA}]$ \\
\hline SV_EM & 10 & $-3 \pm 1$ \\
SV_SRK & 10 & $-2 \pm 1$ \\
HD_EM & 10 & $-2 \pm 1$ \\
HD_SRK & 10 & $-2 \pm 0$ \\
SV_EM & 50 & $-4 \pm 1$ \\
SV_SRK & 50 & $-1 \pm 1$ \\
HD_EM & 50 & $-2 \pm 1$ \\
HD_SRK & 50 & $-1 \pm 1$ \\
SV_EM & 100 & $-2 \pm 1$ \\
SV_SRK & 100 & $-4 \pm 1$ \\
HD_EM & 100 & $-3 \pm 1$ \\
HD_SRK & 100 & $-1 \pm 1$ \\
\hline \hline
\end{tabular}


a) SV model, EM propagator

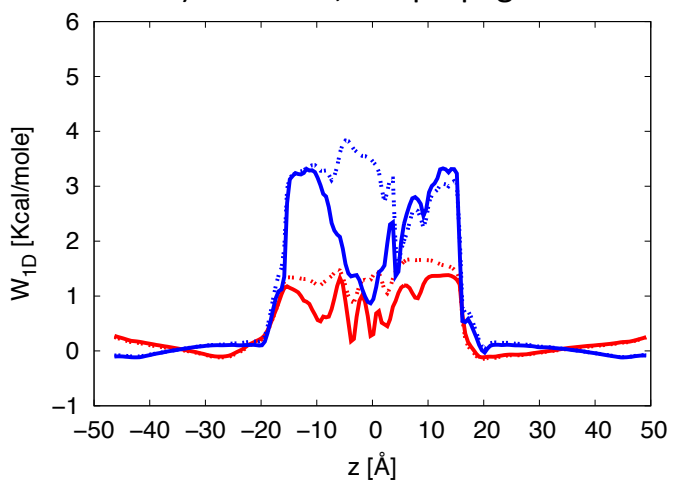

b) SV model, SRK propagator

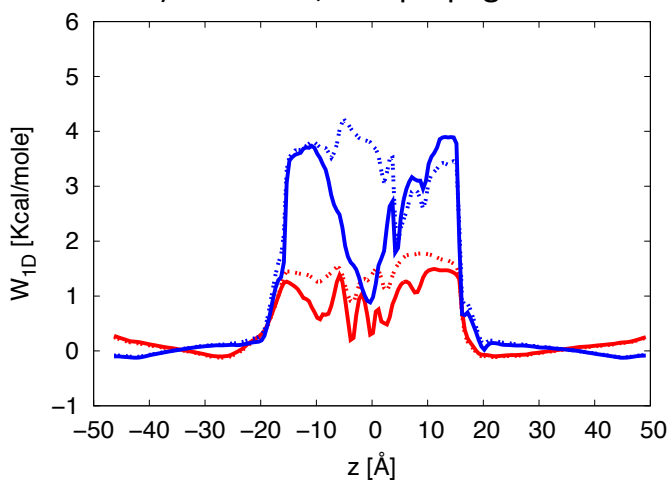

c) HD model, EM propagator

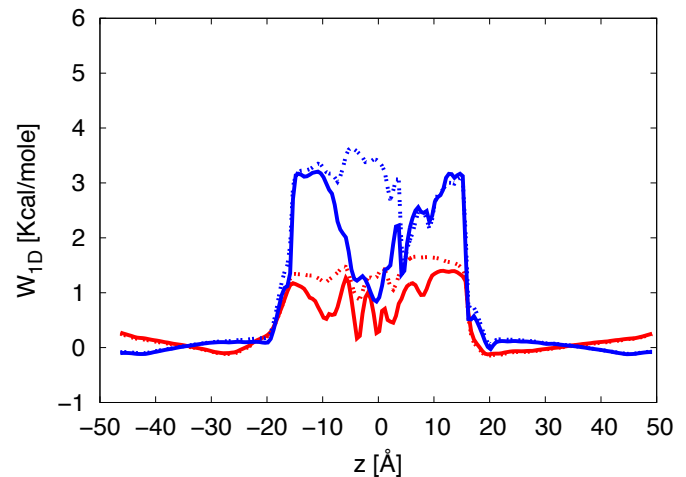

Figure S4: Same as in Figure S3 but using the alternative core-repulsive scheme. To facilite comparison, the multi-ion PMF for the respective original GCMC/BD simulations (see Figure S2) are given as dotted lines.

Table S6: Total average current $(I)$ for original GCMC/BD simulations at equilibrium conditions. The time step is set to $10 \mathrm{fs}$. The switching distances $r_{\text {on }}$ and $r_{\text {off }}$ were set to 8 and $12 \AA$, respectively.

\begin{tabular}{ccc}
\hline \hline & REPULSIVE SCHEME & $I[\mathrm{pA}]$ \\
\hline SV_EM & original & $-5 \pm 1$ \\
SV_SRK & original & $-4 \pm 1$ \\
HD_EM & original & $-2 \pm 1$ \\
HD_SRK & original & $-2 \pm 1$ \\
SV_EM & alternative & $-5 \pm 1$ \\
SV_SRK & alternative & $-4 \pm 1$ \\
HD_EM & alternative & $-2 \pm 0$ \\
HD_SRK & alternative & $-2 \pm 1$ \\
\hline \hline
\end{tabular}


a) SV model, EM propagator

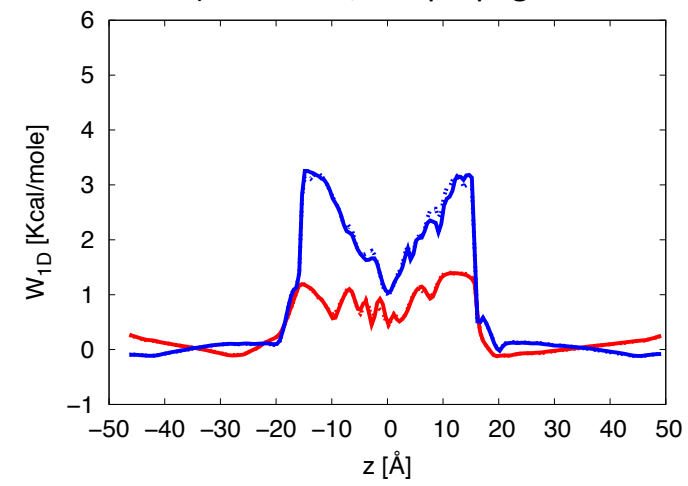

b) SV model, SRK propagator

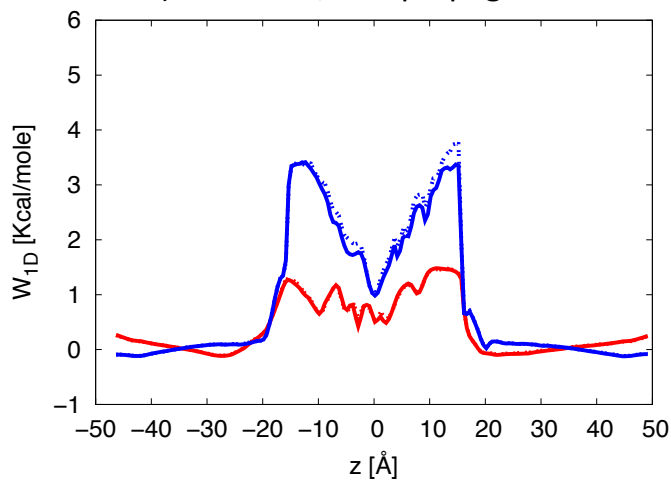

c) HD model, EM propagator

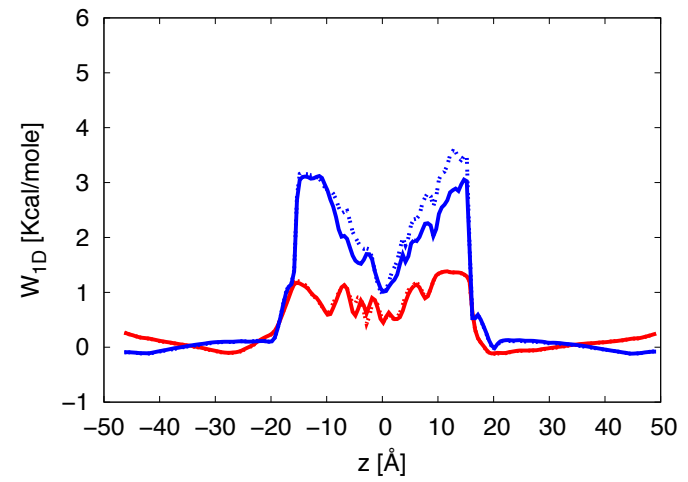

Figure S5: 1D multi-ion average PMF extracted from 10 independent flexible GMC/BD simulations under equilibrium conditions $\left(V_{m p}=0\right)$ using the alternative core-repulsive scheme. The results for the $\mathrm{K}^{+}$ions are represented in red while for the $\mathrm{Cl}^{-}$ions in blue. GCMC/BD simulations using a timestep of $10 \mathrm{fs}$ are plotted in solid lines and as a dotted lines using a time step of $5 \mathrm{fs}$. 
Table S7: Total average current $(I)$ for restrained GCMC/BD simulations at equilibrium conditions. The time step is set to $10 \mathrm{fs}$. The switching distances $r_{o n}$ and $r_{o f f}$ were set to 8 and $12 \AA$, respectively.

\begin{tabular}{ccc}
\hline \hline & REPULSIVE SCHEME & $I[\mathrm{pA}]$ \\
\hline SV_EM & original & $-8 \pm 3$ \\
SV_SRK & original & $-8 \pm 3$ \\
HD_EM & original & $-6 \pm 2$ \\
HD_SRK & original & $-1 \pm 2$ \\
SV_EM & alternative & $-5 \pm 4$ \\
SV_SRK & alternative & $-12 \pm 2$ \\
HD_EM & alternative & $-8 \pm 3$ \\
HD_SRK & alternative & $-4 \pm 3$ \\
\hline \hline
\end{tabular}

Table S8: Total average current $(I)$ for flexible GCMC/BD simulations using the alternative core-repulsive potential scheme at equilibrium conditions. The switching distances $r_{\text {on }}$ and $r_{\text {off }}$ were set to 8 and $12 \AA$, respectively.

\begin{tabular}{ccc}
\hline \hline & $\Delta t[\mathrm{fs}]$ & $I[\mathrm{pA}]$ \\
\hline SV_EM & 10 & $-11 \pm 3$ \\
SV_EM & 5 & $-5 \pm 6$ \\
SV_SRK & 10 & $-4 \pm 2$ \\
SV_SRK & 5 & $-7 \pm 6$ \\
HD_EM & 10 & $-4 \pm 2$ \\
HD_EM & 5 & $-1 \pm 2$ \\
HD_SRK & 10 & $-4 \pm 3$ \\
HD_SRK & 5 & $-10 \pm 4$ \\
\hline \hline
\end{tabular}

and $12 \AA$, respectively, are detailed in Table S6. Moreover, in Table S7 the total average currents for equilibrium simulations described in Section 5.5 of main text are listed where the switching distances $r_{o n}$ and $r_{\text {off }}$ were set to 8 and $12 \AA$, respectively. If one compares these current values with those shown in Table S6, one can observe an slight increase in the current noise. Since the resolution of the current estimate is defined by the timescale of the simulations, this is a consequence of the reduction of the simulation by time an order of magnitude in these simulations (see main text). The total average current for equilibrium simulations described in Section 5.6 of main text is given in Table $\mathrm{S} 8$ where the switching distances $r_{\text {on }}$ and $r_{\text {off }}$ were set to 8 and $12 \AA$, respectively. On can observe an slight increase in the uncertainties when the time step is 5 fs. This a consequence of the simulation time reduction by a factor of two in these simulations.

\section{Molecular Dynamics Simulations}

Molecular dynamics (MD) simulations were performed using the GROMACS 4.6.5 program [9] and the CHARMM36 force field [10,11]. The simulations were carried out using a $2 \mathrm{fs}$ time step at a temperature of $300 \mathrm{~K}$. Short-range electrostatics were calculated with a cutoff of $12 \AA$ whereas long-range electrostatics were treated with the particle-mesh Ewald method [12]. Furthermore, the short range van der Waals interactions were considered up to a distance of $10 \AA$ and a switching function was used to smoothly turn off these interactions at $12 \AA$. All bonds were 


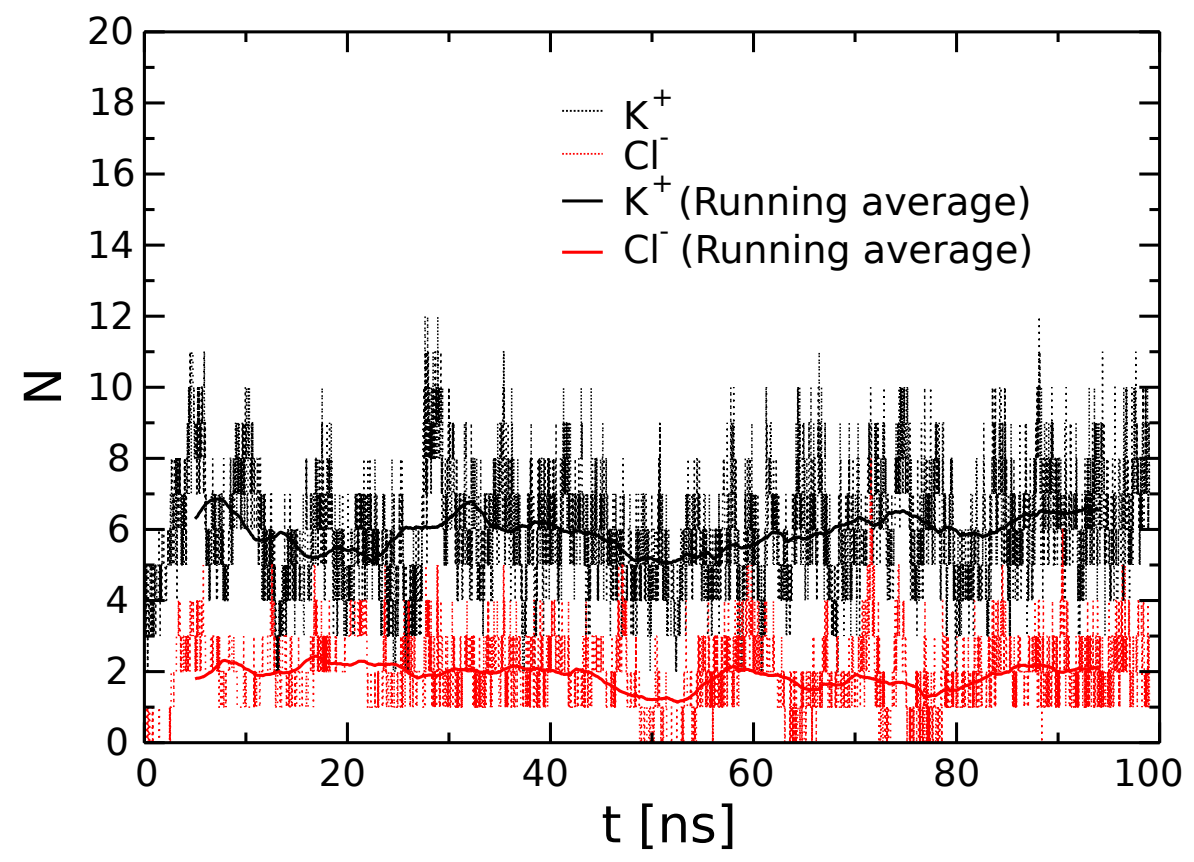

Figure S6: Instantaneous and average number of ions, $N$, in the OmpC channel region vs simulation time from MD simulations. $\mathrm{K}^{+}$ions are represented in black and $\mathrm{Cl}^{-}$ions in red.

constrained using the LINCS algorithm [13].

The crystal structure of the OmpC channel (PDB ID:2J1N) was used as a starting conformation and only one monomer was considered in these simulations. All residues of the protein were assumed to stay in their normal protonation state. The channel axis was aligned along $z$-axis and placed in the center of the 1-Palmitoyl-2-oleoyl-sn-glycero-3phosphoethanolamine (POPE) bilayer. A $300 \mathrm{mM} \mathrm{KCl}$ solution was added using the TIP3P water model [14]. This system was minimized by the steepest descent method and equilibrated in the following steps. First, only lipid molecules were relaxed for 2 ns in NVT ensemble. Thereafter, only protein $\mathrm{C}_{\alpha}$ atoms were restrained for $3 \mathrm{~ns}$ in NVT ensemble. Finally, the system was equilibrated without restraints for $5 \mathrm{~ns}$ in NPT ensemble $(\mathrm{P}=1 \mathrm{~atm})$. To the resulting system, a constant electric field (corresponding to $0.1 \mathrm{~V}$ ) was applied along the $z$-axis of the system. This is a widely adopted method to model the transmembrane potenital across the channel in simulations $[15,16]$. The final production run of $100 \mathrm{~ns}$ was performed with an applied electric field in NVT ensemble. The temperature was set to $300 \mathrm{~K}$ in all steps.

In Fig. S6 the average number of ions in the channel region vs. simulation time are shown. The channel region was defined between -15 and $15 \AA$, i.e., the same region used in GCMC/BD simulations. The total average numbers are $N_{K^{+}}=5.95$ and $N_{C l^{-}}=1.87$ for potassium and chloride ions, respectively. The standard deviations are $\sigma_{K^{+}}=1.64$ and $\sigma_{C^{-}}=1.06$.

The resolution of the procedure for estimating ion conductance is defined by the time scale of the simulation, which should be sufficiently long to collect a statistically significant number of ion permeations. To obtain statistically meaningful numbers of ion permeation events, is a common practice to apply a voltage a few times larger (i.e., in the range of $1 \mathrm{~V}$ ) than the usually applied in biological experiments (i.e., $50-150 \mathrm{mV}$ ) and assume that the voltage difference between experiment and simulation does not introduce a major deviation in the results [17]. Indeed, the 


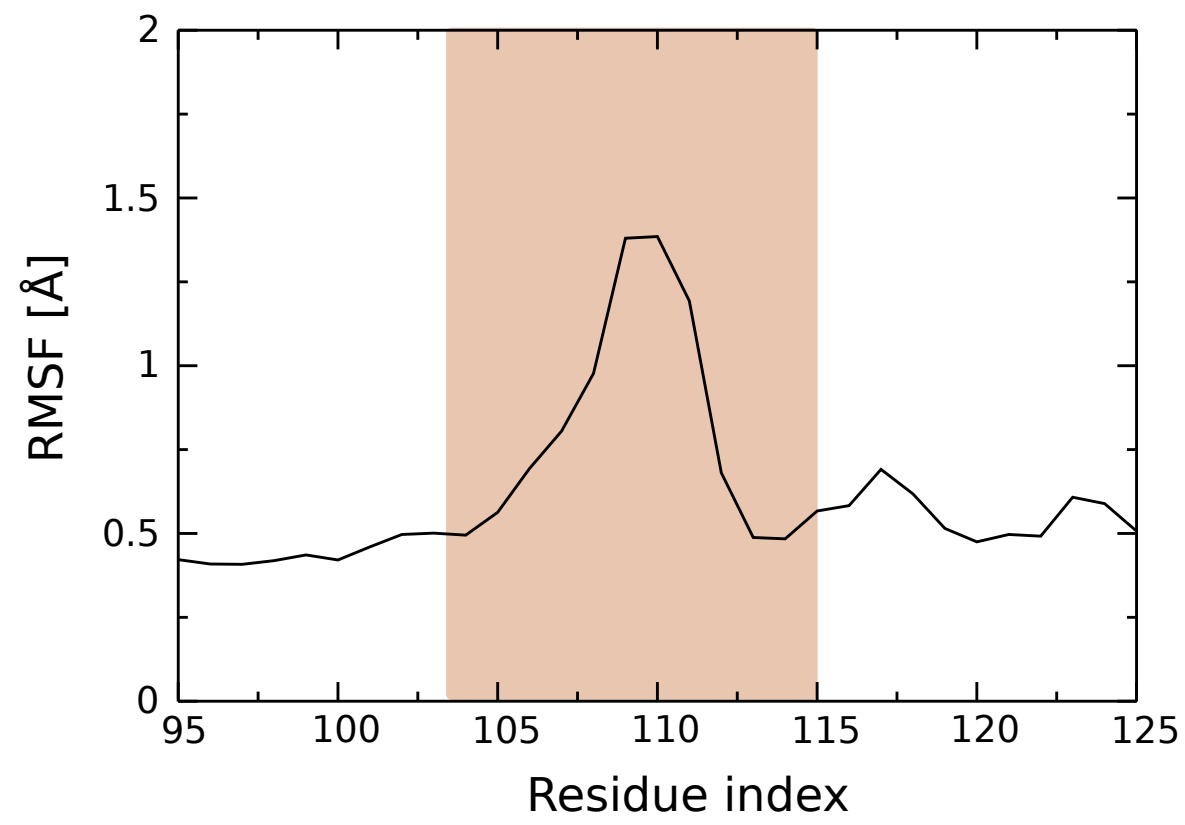

Figure S7: RMSF of the $\mathrm{C}_{\alpha}$ atoms belonging to the residues forming the L3 loop over $100 \mathrm{~ns}$ of the applied-field MD trajectory. All frames of the trajectory are aligned to the starting structure of the simulation. The flexible residues in the L3 loop (see Section 5.6 of the main text), i.e., residues 104 to 114, are highlighted.

main goal of these applied-field MD simulations is to provide a number of ions inside the channel which can be directly compared to that obtained from GCMC/BD simulations. Since the number of ions inside the channel depends on the transmembrane potential, temperature and solution concentration, one should run MD simulations under the same conditions as for the GCMC/BD simulations. The time scales of the present MD simulations are, however, not sufficient to obtain accurate values for conductances and therefore we refrain from trying to extract these values from the present simulations.

In Fig. S7 the root-mean square fluctuations (RMSF) of the $\mathrm{C}_{\alpha}$ atoms belonging to the residues forming the L3 loop are shown over $100 \mathrm{~ns}$ of an applied-field MD trajectory.

\section{References}

[1] B. Roux, Biophys. J. 73, 2980 (1997).

[2] W. Im, D. Beglov and B. Roux, Comp. Phys. Comm. 111, 59 (1998).

[3] W. Im and B. Roux, J. Chem. Phys. 115, 4850 (2001).

[4] W. Im, S. Bernèche, and B. Roux, J. Chem. Phys. 114, 2924 (2001).

[5] J.-P Ryckaert, G. Ciccotti, H. J. C. Berendsen, J. Comput. Phys. 23, 327 (1977).

[6] P. L'Ecuyer, Oper. Res. 47, 159 (1999).

[7] P. L'Ecuyer, ACM Transactions on Mathematical Software 33, 22 (2007). 
[8] R. E. Odeh and J. O. Evans, App. Stat. 23, 96 (1974).

[9] B. Hess, C. Kutzner, D. van der Spoel, E. Lindahl, J. Chem. Theory Comput. 4, 435 (2008).

[10] A. D. MacKerell, M. Feig, C. L. Brooks, J. Comput. Chem. 25, 1400 (2004).

[11] J. B. Klauda, R. M. Venable, J. A. Freites, J. W. OConnor, D. J. Tobias, C. Mondragon Ramirez, I. Vorobyov, A. D. MacKerell Jr, R. W. Pastor, J. Phys. Chem. B 114, 7830 (2010).

[12] T. Darden, D. York, L. Pedersen, J. Chem. Phys. 98, 10089 (1993).

[13] B. Hess, H. Bekker, H. J. C. Berendsen, G. E. M. F. Johannes, J. Comput. Chem. 18, 1463 (1997).

[14] W. L. Jorgensen, J. Chandrasekhar, J. D. Madura, R. W. Impey, M. L. Klein, J. Chem. Phys. 79, 926 (1983).

[15] A. Aksimentiev, K. Schulten, Biophys. J. 88, 3745 (2005).

[16] J. Gumbart, F. Khalili Araghi, M. Sotomayor, B. Roux, Biochimica et Biophysica Acta (BBA) 1818, 294 (2012).

[17] N. Modi, M. Winterhalter and U. Kleinekathöfer, Nanoscale 4, 6166 (2012). 